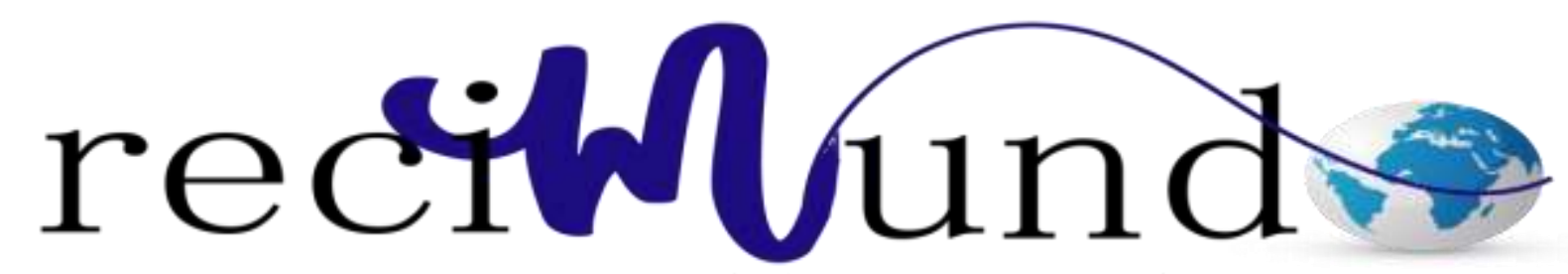

Revista Cientifica Mundo de la Investigación y el Conocimiento

\author{
María Judith Naranjo Escobar ${ }^{\text {a }}$; Cristhian Jacinto Macas Quevedo ${ }^{\text {b; }}$ María José

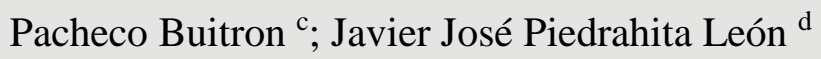

Obstrucción del conducto nasolagrimal: abordaje quirúrgico endonasal versus externo

Nasolacrimal duct obstruction: endonasal versus external surgical approach

Revista Científica Mundo de la Investigación y el Conocimiento. Vol. 3 núm.3, septiembre, ISSN: 2588-073X, 2019, pp. 509-526

DOI: $10.26820 /$ recimundo/3.(3).septiembre.2019.509-526

URL: http://recimundo.com/index.php/es/article/view/534

Código UNESCO: 3205 Medicina Interna

Tipo de Investigación: Artículo de Revisión

Editorial Saberes del Conocimiento

Recibido: 15/05/2019

Aceptado: 23/06/2019

Publicado: 30/09/2019

Correspondencia: mariajudithnaranjo@gmail.com
a. Médico; Investigador Independiente; Guayaquil, Ecuador; mariajudithnaranjo@gmail.com
b. Médico; Investigador Independiente; Guayaquil, Ecuador; drcristhianmacas@ gmail.com
c. Médico; Investigador Independiente; Guayaquil, Ecuador; majopabui@ hotmail.com
d. Médico; Investigador Independiente; Guayaquil, Ecuador; javier.p.leon@gmail.com 


\section{Obstrucción del conducto nasolagrimal: abordaje quirúrgico endonasal versus externo}

Vol. 3, núm. 3., (2019)

María Judith Naranjo Escobar; Cristhian Jacinto Macas Quevedo; María José Pacheco Buitron; Javier José Piedrahita León

\section{RESUMEN}

A lo largo del sistema de drenaje lagrimal existen distintas estructuras membranosas que generalmente se abren de manera espontánea poco antes, o inmediatamente después, del nacimiento. Esta condición es un bloqueo parcial o completo en el conducto que transporta las lágrimas desde la superficie del ojo hasta la nariz. Aproximadamente 1 de cada 25 bebés nace con los conductos lagrimales obstruidos. Esto generalmente ocurre porque el extremo inferior del conducto lagrimal no se abre antes de que nazca el bebé. El sistema de drenaje naso lagrimal se desarrolla a partir de la invaginación del ectodermo superficial que se origina en la fisura nasoóptica. Las obstrucciones altas (proximales) que se localizan en el punto lagrimal, pueden ser tratadas con colirios de cortisona, en el caso de que la obstrucción sea por edema (hinchazón) o bien sea por medio de la técnica quirúrgica de punto plastia con la que se ensancha el orificio de entrada. Un bloqueo en el conducto naso lagrimal puede aumentar el riesgo de infecciones oculares. Cualquier persona que presente derrame de lágrimas sobre las mejillas necesita un examen, ya que una de las posibles causas es un tumor.

Palabras Claves: Obstrucción; Bloqueo; Nasolagrimal; Epifora; Dacriocistorrinostomia. 


\title{
Obstrucción del conducto nasolagrimal: abordaje quirúrgico endonasal
}

versus externo

Vol. 3, núm. 3., (2019)

María Judith Naranjo Escobar; Cristhian Jacinto Macas Quevedo; María José Pacheco Buitron; Javier José Piedrahita León

\begin{abstract}
Along the tear drainage system there are different membranous structures that generally open spontaneously shortly before, or immediately after birth. This condition is a partial or complete blockage in the duct that carries tears from the surface of the eye to the nose. Approximately 1 in 25 babies is born with clogged tear ducts. This usually occurs because the lower end of the tear duct does not open before the baby is born. The lacrimal naso drainage system develops from the invagination of the superficial ectoderm that originates in the naso-optic fissure. The high (proximal) obstructions that are located in the tear point can be treated with cortisone eye drops, in the event that the obstruction is due to edema (swelling) or by means of the surgical technique of plastia point with which the entrance hole widens. A blockage in the tear duct can increase the risk of eye infections. Anyone with tears on the cheeks needs an exam, since one of the possible causes is a tumor.
\end{abstract}

Key Words: Obstruction; Blockage; Nasolacrimal; Epiphora; Dacryocystorrinostomy. 


\section{Obstrucción del conducto nasolagrimal: abordaje quirúrgico endonasal versus externo}

Vol. 3, núm. 3., (2019)

María Judith Naranjo Escobar; Cristhian Jacinto Macas Quevedo; María José Pacheco Buitron; Javier José Piedrahita León

\section{Introducción.}

A lo largo del sistema de drenaje lagrimal existen distintas estructuras membranosas que generalmente se abren de manera espontánea poco antes, o inmediatamente después, del nacimiento.

La causa más común de obstrucción del conducto nasolagrimal se debe a un déficit en la canalización de la porción distal del conducto nasolagrimal, con la adición de un defecto membranoso a nivel de la válvula de Hasner.

La sintomatología se presenta antes del mes de edad en el $80 \%$ de los casos y consiste típicamente en epifora y secreción que se acumula en los parpados; la presión suave sobre el saco lagrimal produce reflujo de material purulento por el punto lagrimal. Los síntomas pueden estar presentes al nacimiento, aunque generalmente suelen manifestarse a las pocas semanas de edad cuando aumenta la producción de lágrimas. La obstrucción puede presentarse en ambos ojos, aunque habitualmente es unilateral.

El sondaje del conducto lagrimal, en muchos estudios, se ha reportado con un alto índice de éxito, siempre y cuando se realice antes del primer año de vida; algunos autores mencionan que el sondaje temprano disminuye la fibrosis secundaria debida a inflamación crónica. El procedimiento es realizado bajo anestesia general o sedación. La punta de la sonda puede pasarse varias veces a través de la zona obstruida para optimizar la maniobra. Sabiendo que esta es una condición clínica bastante común, se debe tener en cuenta que existen también varias complicaciones de carácter genético o adquirido. Y tener el conocimiento de los otros factores 


\section{Obstrucción del conducto nasolagrimal: abordaje quirúrgico endonasal \\ versus externo}

Vol. 3, núm. 3., (2019)

María Judith Naranjo Escobar; Cristhian Jacinto Macas Quevedo; María José Pacheco Buitron; Javier José Piedrahita León

que podrían llevar al desarrollo de esta condición. (Gonzàles \& Pèrez, 2014)

\section{Materiales y Métodos.}

Las herramientas y materiales que se conciben para el desarrollo de este trabajo investigativo son los siguientes: computadores personales con conexión a internet y un cúmulo de contenidos científico-académicos diversos, con los que se busca aportar información de calidad y actualizada con respecto a la Obstrucción del conducto naso lagrimal. Se escoge un diseño bibliográfico, en el marco de una metodología de revisión sistemática.

Esta investigación se enfoca en la búsqueda y revisión sistemática de literatura científico-académica seleccionada, que por una parte, está disponible determinadas bases de datos, entre las que figuran: MedlinePlus, PubMed, Biblioteca Virtual de la Salud (BVS), SciELO, Dialnet y ELSEVIER, Cochrane, entre otras; representando todo ello parte esencial del proceso investigativo y de comprensión que facilita la síntesis de la mejor evidencia disponible, y a su vez representando todo ello uno los resultados esperados.

Principalmente se realiza una búsqueda aleatoria y consecutiva en las mencionadas bases de datos, usando las expresiones "obstrucción naso lagrimal", "bloqueo lagrimal", "la dacriocistorrinostomia" y "epifora", lo que aproximadamente resultó en miles de registros bibliográficos. Luego éstos se filtran en base a criterios de: idioma español, relevancia, correlación temática y fecha de publicación en años anteriores, sin descartar por tipo de material bibliográfico; es decir, se escogen títulos de artículos científicos, ensayos, revisiones sistemáticas, tesis de grado, posgrado y doctorado, noticias científicas, entre otros documentos e 


\section{Obstrucción del conducto nasolagrimal: abordaje quirúrgico endonasal versus externo}

Vol. 3, núm. 3., (2019)

María Judith Naranjo Escobar; Cristhian Jacinto Macas Quevedo; María José Pacheco Buitron; Javier José Piedrahita León

información de interés científico y académico.

Seguidamente, se efectúa la selección y clasificación de la literatura científica académica disponible, aplicando, en términos generales, los criterios antes mencionados, siendo a partir de allí que el equipo investigador le da la correspondiente lectura crítica y análisis de toda esa evidencia científica, lo que resultó consecutiva y consensuadamente en el fundamento de las ideas y planteamientos aquí plasmados.

\section{Resultados.}

La obstrucción del conducto naso lagrimal es un bloqueo del conducto lagrimal que ocurre cuando el sistema de drenaje de lágrimas es parcial o totalmente obstruido. Las lágrimas no pueden ser drenadas normalmente, causando ojos acuosos, irritados o crónicamente infectados. La mayoría de las lágrimas provienen de las glándulas lagrimales, situadas encima de cada ojo. Las lágrimas fluyen hacia la superficie del ojo para lubricarlo y protegerlo, y drenan a través de unos pequeños orificios (puntos lagrimales) en las esquinas de los párpados superiores e inferiores. Las lágrimas luego viajan a través de unos pequeños canales en los párpados (canalículos lagrimales) al saco lagrimal, situado donde el párpado y la nariz se unen, y continúan a través del conducto nasolagrimal antes de llegar a la nariz, donde se evaporan o son reabsorbidas. (American Academy of Ophthalmology, 2015)

En los niños, es posible que este conducto no esté completamente desarrollado al nacer. Puede estar cerrado o cubierto por una delgada película, lo que causa un bloqueo parcial. En los adultos, el conducto puede resultar dañado por una infección, una lesión o un tumor. (Medline 


\section{Obstrucción del conducto nasolagrimal: abordaje quirúrgico endonasal}

versus externo

Vol. 3, núm. 3., (2019)

María Judith Naranjo Escobar; Cristhian Jacinto Macas Quevedo; María José Pacheco Buitron; Javier José Piedrahita León

Plus, 2016)

Tipos del lagrimal obstruido Adquirido

Este tipo es el común. Se produce cuando el lagrimal obstruido afecta a personas adultas por el paso del tiempo. (Oftalvist, 2017)

\section{Congénito}

Existe una forma congénita, en el recién nacido, que suele resolverse por sí sola en un 90\% de los casos antes del año. (Oftalvist, 2017)

El bebé presenta un lagrimeo constante, a menudo, acompañado de muchas secreciones mucopurulentas, lagrimeo constante, legañas enormes e inflamación del párpado inferior. Es lo que se llama estenosis o estrechamiento de los conductos lagrimales. Una afección que se debe a la inmadurez del sistema nasolagrimal.

Como tratamiento, es necesario limpiarle bien los ojos al menos tres veces al día siguiendo las indicaciones de un oftalmólogo pediatra con unas toallitas especiales y masajearle el ángulo interno del ojo ejerciendo presión suavemente hacia abajo con el dedo.

Si no se resuelve al año será necesario operar con un sondaje. Un procedimiento quirúrgico sencillo de no más de 15 minutos que no resulta dolorosa para el bebé y se realiza bajo sedación y colirio anestésico. (Oftalvist, 2017) 


\section{Obstrucción del conducto nasolagrimal: abordaje quirúrgico endonasal versus externo}

Vol. 3, núm. 3., (2019)

María Judith Naranjo Escobar; Cristhian Jacinto Macas Quevedo; María José Pacheco Buitron; Javier José Piedrahita León

\section{Síntomas:}

Los signos y síntomas de obstrucción del conducto lagrimal incluyen:

$\circ \quad$ Lagrimeo excesivo

○ Enrojecimiento de la parte blanca del ojo

○ Infección o inflamación ocular recurrente (conjuntivitis)

○ Inflamación dolorosa cerca de la esquina interior del ojo

○ Formación de costras en los párpados

- Secreción de mucosidad o pus de los párpados y la superficie del ojo

○ $\quad$ Visión borrosa (Mayo Clinic, 2016)

Signos de obstrucción de los conductos naso lagrimales

Los bebés con este tipo de obstrucción suelen presentar síntomas entre el nacimiento y la duodécima semana de vida, aunque es posible que no se detecte este problema hasta que el niño desarrolle una infección ocular. Los signos más frecuentes son el exceso de lagrimeo, incluso en ausencia de llanto (lo que se conoce como epifora). También puede producirse pus en la comisura del ojo, o que el niño se despierte con los párpados y/o pestañas cubiertos de legañas.

Los niños con obstrucción de los conductos naso lagrimales pueden desarrollar una infección en el saco lagrimal llamada dacriocistitis. Los signos incluyen el enrojecimiento de la 


\section{Obstrucción del conducto nasolagrimal: abordaje quirúrgico endonasal}

versus externo

Vol. 3, núm. 3., (2019)

María Judith Naranjo Escobar; Cristhian Jacinto Macas Quevedo; María José Pacheco Buitron; Javier José Piedrahita León

comisura interna del ojo y una ligera molestia e hinchazón o abultamiento en el lado de la nariz.

Abecés se da el caso de que algunos lactantes nazcan con un quiste en el saco lagrimal, que provoca un abultamiento azul llamado dacriocistocele cerca de la comisura interna del ojo.

Aunque esta afección debe ser controlada por un médico de forma muy atenta, no siempre evoluciona a una infección y se puede tratar en casa con masaje firme y observación. Si se infectara, se necesitarían antibióticos por vía tópica (aplicados sobre la piel).

De todos modos, en algunas infecciones, el bebé puede tener que ingresar en un hospital, donde le administrarán antibióticos por VI (vía intravenosa), seguidos de una operación donde le desbloquea el conducto con una sonda. (Salvin, 2015)

Causas de obstrucción del conducto lagrimal

Aproximadamente 1 de cada 25 bebés nace con los conductos lagrimales obstruidos. Esto generalmente ocurre porque el extremo inferior del conducto lagrimal no se abre antes de que nazca el bebé. A veces un conducto lagrimal se obstruye más adelante en la vida. (Abou Tkids Health, 2009)

Ya que en algunos bebés no se encuentra desarrollado por completo el conducto nasolagrimal al momento de nacer, (esto se conoce como dacriostenosis) pero la obstrucción generalmente desaparece hacia los 6 meses, sin necesidad de tratamiento. (Provisu, 2016) 


\section{Obstrucción del conducto nasolagrimal: abordaje quirúrgico endonasal versus externo}

Vol. 3, núm. 3., (2019)

María Judith Naranjo Escobar; Cristhian Jacinto Macas Quevedo; María José Pacheco Buitron; Javier José Piedrahita León

Obstrucción congénita del conducto nasolagrimal

La causa más frecuente de epifora en el lactante es la obstrucción del conducto nasolagrimal (OCNL), siendo la incidencia en el mundo occidental de un 6\%. El sistema de drenaje nasolagrimal se desarrolla a partir de la invaginación del ectodermo superficial que se origina en la fisura naso-óptica. Su canalización comienza en la zona media del sistema y continúa de forma ascendente y descendente. El proceso suele completarse para el final del noveno mes de gestación. Si la canalización resulta incompleta, se produce una obstrucción en la salida de las lágrimas. El lugar más común de obstrucción es la parte más distal del conducto nasolagrimal, persistiendo una obstrucción membranosa en la válvula de Hasner. Cualquier bloqueo aquí o en algún otro nivel del sistema de drenaje lagrimal causará una acumulación de lágrimas en el ojo.

Clínicamente, los lactantes con una OCNL (obstrucción del conducto nasolagrimal) se presentan con epifora o lagrimeo a las 2-6 semanas de vida, secreción mucosa que se acumula en los márgenes de los párpados y en las pestañas y dermatitis secundaria. La conjuntivitis es causada por una sobre infección de las lágrimas estancadas y de la secreción mucosa (con secreción mucopurulenta abundante, hiperemia y molestias oculares) y, en menor grado, celulitis palpebral. Esta misma sobreinfección del contenido del saco lagrimal causará una dacriocistitis, presentándose con dolor, tumefacción, rubor y calor en el área del canto interno, pudiéndose palpar un saco lagrimal edematoso. La OCNL es bilateral en aproximadamente 1/3 de los casos.

La mitad de todos los casos se resolverá espontáneamente para los 6 meses de edad, disminuyendo esta incidencia drásticamente después de los 13 meses de edad. (Lawand, 2013) 


\section{Obstrucción del conducto nasolagrimal: abordaje quirúrgico endonasal \\ versus externo}

Vol. 3, núm. 3., (2019)

María Judith Naranjo Escobar; Cristhian Jacinto Macas Quevedo; María José Pacheco Buitron; Javier José Piedrahita León

¿Qué tratamientos existen actualmente?

Las obstrucciones altas (proximales) que se localizan en el punto lagrimal, pueden ser tratadas con colirios de cortisona, en el caso de que la obstrucción sea por edema (hinchazón) o bien sea por medio de la técnica quirúrgica de punto plastia con la que se ensancha el orificio de entrada. (Barraquer, 2018)

\section{Tratamiento de los conductos obstruidos}

Como ya se dicho antes los niños que tienen los conductos naso lagrimales obstruidos a menudo se pueden tratar en casa. Es posible que el pediatra del niño o un oftalmólogo pediátrico recomiende masajearle el ojo afectado varias veces al día durante un par de meses. Antes de practicarle el masaje, se debe higienizar las manos con agua y jabón. Colocar el dedo índice en la parte lateral de la nariz del bebé y realizar un masaje firme hacia abajo hasta el extremo inferior de la nariz. También se pueden aplicar compresas calientes sobre el ojo para favorecer el drenaje y aliviarle cualquier molestia que pueda tener.

Si él bebe desarrolla una infección como consecuencia de la obstrucción de los conductos naso lagrimal, el pediatra le recetará gotas oculares antibióticas o pomada ocular antibiótica para tratar la infección. Es de suma importancia recordar que los antibióticos no eliminarán la obstrucción. En cuanto remita la infección, podrá seguir masajeando los conductos naso lagrimales del bebe tal y como le haya indicado el pediatra. (Lehman, 2008) 


\section{Obstrucción del conducto nasolagrimal: abordaje quirúrgico endonasal versus externo}

Vol. 3, núm. 3., (2019)

María Judith Naranjo Escobar; Cristhian Jacinto Macas Quevedo; María José Pacheco Buitron; Javier José Piedrahita León

La dacriocistorrinostomía

Es un procedimiento quirúrgico generalmente realizado para tratar la mayoría de los casos de conductos lagrimales bloqueados. Esta técnica traza una nueva variante (ruta) de drenaje de lágrimas a través de la nariz permitiendo que éstas salgan de nuevo con normalidad, creando así una conexión entre el saco lagrimal y la nariz. Por medio de un bypass, la nueva variante de drenaje se salta el conducto que desemboca en la nariz (llamado conducto nasolagrimal, en el que suele estar la obstrucción). Normalmente, la intubación o canulización es colocada en la nueva variante durante la recuperación, y es removida unos tres o cuatro meses después de haberse realizado la cirugía. Los pasos a seguir en éste procedimiento varían de acuerdo a la gravedad del bloqueo en el conducto lagrimal de cada individuo.

Dependiendo del tipo de obstrucción, el cirujano puede recomendar la creación de una variante completamente nueva desde la esquina interna del ojo (puntos lagrimales) hasta la nariz, saltando por completo el sistema de drenaje lagrimal. A ésta reconstrucción del sistema de drenaje lagrimal se le llama conjuntivodacriocistorrinostomía. (American Academy of Ophthalmology , 2012)

Obstrucción de la via lagrimal tratada mediante stent lacrimonasal

Se logró realizar el implante de la prótesis con éxito en 120 de los 125 casos (96\% del total). Las principales complicaciones intraoperatorias fueron: Dolor 5,83\% (7 casos), inflamación palpebral 5\% (6 pacientes), creación de falsa vía (diagnosticada clínicamente) $0,83 \%$ ( 1 caso) y epistaxis (con tratamiento por parte de Otorrinolaringología) $0,83 \%$ (1 caso). 


\section{Obstrucción del conducto nasolagrimal: abordaje quirúrgico endonasal \\ versus externo}

Vol. 3, núm. 3., (2019)

María Judith Naranjo Escobar; Cristhian Jacinto Macas Quevedo; María José Pacheco Buitron; Javier José Piedrahita León

La principal complicación postoperatoria fue mucocele 7,5\% (9 casos).

Los resultados de los stents implantados tras un año de estudio fueron: desaparición de la epifora y los otros síntomas en 82 pacientes $(68,3 \%)$ y persistencia en $38(31,7 \%)$.

En los casos en que el stent no logró solucionar la obstrucción de la vía lagrimal, el tiempo medio de fallo fue de 178 días. Los fallos de los implantes se han dividido entre los sucedidos en el primer mes ( 2 casos; $5,26 \%$ ), segundo mes ( 8 casos; $21,05 \%)$, los ocurridos entre el tercer y sexto mes (17 pacientes; 44,73\%), los que fallaron en el segundo semestre tras la intervención ( 5 casos; $13,5 \%$ ) y los que sucedieron pasado el primer año (6 pacientes; $15,78 \%)$.

En los casos en que la técnica no dio resultado el tratamiento se realizó mediante una dacriocistorrinostomía en 22 enfermos (57,89\%), dacriocistectomía en 5 (13,15\%), dacriointubación cerrada en $6(15,78 \%)$ y punto plastia en $5(13,15 \%)$.

Tras analizar los stents en los casos de mal funcionamiento, se encontró tejido de granulación en el $63,15 \%$ y material mucoide en el $36,84 \%$, porcentaje muy similar a los encontrados en otras series.

La relativa sencillez en la implantación, las pocas complicaciones intra y postoperatorias que produce y la buena aceptación por parte de los pacientes, ya que no deja cicatriz y no es necesario el ingreso hospitalario, además de la facilidad para su extracción si es necesario, hace que la implantación del stent lacrimonasal de poliuretano sea una técnica recomendable para muchos de los pacientes afectos de obstrucción de vía lagrimal, sobre todo para aquellos que tengan la obstrucción localizada en el conducto lacrimonasal o en la unión del conducto al saco 


\section{Obstrucción del conducto nasolagrimal: abordaje quirúrgico endonasal versus externo}

Vol. 3, núm. 3., (2019)

María Judith Naranjo Escobar; Cristhian Jacinto Macas Quevedo; María José Pacheco Buitron; Javier José Piedrahita León

lagrimal de dicho conducto y para los pacientes con sacos de tamaño medio o grande. Sin embargo, debe hacerse notar que su efectividad a largo plazo [con una media de resultados positivos de un $75 \%$ y unos resultados parciales que varían entre el 5 y el $89 \%$ es menor que en otras técnicas, sobre todo la dacriocistorrinostomía endonasal, con un 97\%, la dacriocistorrinostomía externa [que nos ofrece unos resultados de éxito entre el 89 y el 93,2\% con una media del 91,86\%], similar al stent de plástico (79\%) y superior a técnicas como el stent metálico [25\% de efectividad] o la dilatación con balón (45\%); y también que está contraindicada en pacientes que tengan la obstrucción en el canalículo superior, inferior o en el común; así como en sacos muy estrechos. Por tanto, debemos ser muy cuidadosos en la elección de los casos en los que puede practicarse esta técnica. (Soler, Castillo, De Gregorio, \& Bescós, 2003)

\section{Dacriocistorrinostomía transcanalicular con láser diodo}

La intervención quirúrgica se consideraba un éxito si desaparecía la epifora y en el test de Jones I se comprobaba directamente con endoscopio la tinción fluoresceínica del nuevo ostium quirúrgico a los 4 meses de la cirugía (2 meses después de retirar los tubos de silicona).

De un total de cuarenta y tres pacientes, treinta y nueve presentaron ausencia de epifora con test de Jones I positivo.

Dos pacientes presentaron epifora ocasional y secreción mucosa con irrigación permeable con test de Jones I negativo y test de Jones II con control video endoscópico directo positivo y uno obstrucción del canalículo inferior por fibrosis iatrogénica a los dos meses de la retirada de 


\section{Obstrucción del conducto nasolagrimal: abordaje quirúrgico endonasal \\ versus externo}

Vol. 3, núm. 3., (2019)

María Judith Naranjo Escobar; Cristhian Jacinto Macas Quevedo; María José Pacheco Buitron; Javier José Piedrahita León

la intubación, correspondiendo estos casos a pacientes con obstrucción idiopática del conducto nasolagrimal. En estos tres casos se objetivó epifora residual frente a agresiones externas (aire, frío, humo, entre otros) y necesidad de secado entre dos y cuatro veces al día (grado 2 de escala de Munk).

Una paciente con antecedentes de dacriocistitis aguda presentó un cierre completo del nuevo ostium.

En un caso no se pudo intubar la nueva vía por pésima colaboración del paciente, manteniéndose esta permeable en los controles postoperatorios.

Como complicación postquirúrgica en una paciente en la que se utilizaron pulsos de $20 \mathrm{~W}$ de potencia, presentó edema y eritema palpebral en región cantal interna a las 72 horas de la cirugía que se resolvió sin necesidad de tratamiento.

En una paciente se retiró la intubación bicanalicular a los 20 días de la cirugía tras la extrusión del nudo por la fosa nasal debido a un estornudo. La vía permaneció permeable en los controles postquirúrgicos.

Un caso presentó laceración de los puntos lagrimales y uno epitelización nasal de los tubos de silicona, correspondiendo a nuestros primeros casos, en los que se intentó mantener la intubación de la nueva vía por más de dos meses. La media de seguimiento postoperatorio fue de 20,3 meses (rango 4-38 meses). Todos los pacientes retornaron a su actividad normal a las 48 horas. (Alañón F. , Alañón, Martínez, \& Cárdenas, 2004) 


\section{Obstrucción del conducto nasolagrimal: abordaje quirúrgico endonasal versus externo}

Vol. 3, núm. 3., (2019)

María Judith Naranjo Escobar; Cristhian Jacinto Macas Quevedo; María José Pacheco Buitron; Javier José Piedrahita León

Tratamiento de la obstrucción congénita de la vía lagrimal mediante balón catéter, intubación monocanalicular y control endoscópico

Técnicamente pudo finalizarse el procedimiento en el 100\% de los pacientes. Todos los pacientes fueron estudiados más de 12 meses después de la cirugía (rango 13 a 48 meses).

El 84,52\% tuvo buenos resultados; los 7,73\%, aceptables, y los 7,73\%, malos. Un caso presentó erosión corneal secundaria a la extrusión del tubo monocanalicular a las 2 semanas, que se resolvió tras su retirada y tratamiento médico tópico lubricante y antibiótico; la vía lagrimal permaneció permeable.

En 3 casos hubo una rotación del balón, fácilmente identificable al estar teñido el suero, con el que se realizó el inflado, por roce con la sonda nasal al realizar el aspirado de secreciones mucosas. Las observaciones más relevantes de este trabajo estriban en que todos los casos mejoraron respecto al estadio previo, no teniendo que recurrir a realizar una dacriocistorrinostomía en ningún caso. (Alañón M. , Alañón, Martínez, \& Cárdenas, 2009)

\section{Conclusiones.}

En este estudio se encontró que el porcentaje de éxito total es del 35\% en pacientes con diagnóstico de dacrioestenosis congénita mayores de un año de edad, tratado mediante un sondaje de vía lagrimal. La distribución de los casos con éxito y fracaso no presentó una asociación con la edad en este grupo de pacientes. (Gonzàles \& Pèrez, 2014). Un bloqueo en el conducto naso lagrimal puede aumentar el riesgo de infecciones oculares. Cualquier persona que presente derrame de lágrimas sobre las mejillas necesita un examen, ya que una de las posibles 


\section{Obstrucción del conducto nasolagrimal: abordaje quirúrgico endonasal \\ versus externo}

Vol. 3, núm. 3., (2019)

María Judith Naranjo Escobar; Cristhian Jacinto Macas Quevedo; María José Pacheco Buitron; Javier José Piedrahita León

causas es un tumor. (Hoy Digital, 2016)

\section{Bibliografía.}

Abou Tkids Health. (2009). Obstrucción del conducto lagrimal. Canada: autor. Recuperado el 12 de 09 de 2019

Alañón, F., Alañón, M., Martínez, A., \& Cárdenas, M. (2004). Dacriocistorrinostomía transcanalicular con láser diodo. Archivos de la Sociedad Española de Oftalmología, 79(7), 325-330. Recuperado el 12 de 09 de 2019, de http://scielo.isciii.es/scielo.php?script=sci_arttext\&pid=S0365-66912004000700005

Alañón, M., Alañón, F., Martínez, A., \& Cárdenas, M. (Noviembre de 2009). Tratamiento de la obstrucción congénita de la vía lagrimal mediante balón catéter, intubación monocanalicular y control endoscópico. Acta Otorrinolaringologica Española, 60(6), 409-414. doi:10.1016/j.otorri.2009.06.006

American Academy of Ophthalmology . (20 de Enero de 2012). American Academy of Ophthalmology . Recuperado el 12 de 09 de 2019, de American Academy of Ophthalmology : https://www.aao.org/salud-ocular/enfermedades/obstruccion-delconducto-lagrimal-tratamiento

American Academy of Ophthalmology. (01 de Marzo de 2015). American Academy of Ophthalmology. Recuperado el 12 de 09 de 2019, de American Academy of Ophthalmology: https://www.aao.org/salud-ocular/enfermedades/obstruccion-delconducto-lagrimal

Barraquer. (2018). Tratamientos para la obstrucción del lagrimal. Barcelona, España: autor. Recuperado el 12 de 09 de 2019

Gonzàles, J., \& Pèrez, J. (Abril-Junio de 2014). Sondaje de vía lagrimal después del año de edad para el tratamiento de la dacrioestenosis congénita. Revista mexicana de oftalmologia, 88(2), 61-66. doi:10.1016/j.mexoft.2013.10.003

Hoy Digital. (2016). La obstrucción del conducto lagrimal. anto Domingo, República Dominicana: autor. Recuperado el 13 de 09 de 2019

Lawand, T. (2013). Patología congénita de la vía lagrimal y patología palpebral. Pediatria integral, 17(7), 463-476. Recuperado el 12 de 09 de 2019, de https://www.pediatriaintegral.es/wp-content/uploads/2013/xvii07/01/463476\%20Patol\%20palpebral.pdf 


\section{Obstrucción del conducto nasolagrimal: abordaje quirúrgico endonasal versus externo}

Vol. 3, núm. 3., (2019)

María Judith Naranjo Escobar; Cristhian Jacinto Macas Quevedo; María José Pacheco Buitron; Javier José Piedrahita León

Lehman, S. (Octubre de 2008). Rady Children. Recuperado el 12 de 09 de 2019, de Rady Children: https://www.rchsd.org/health-articles/obstruccin-y-ciruga-del-conductonasolagrimal/

Mayo Clinic. (2016). Lagrimal bloqueado. Minnesota, United States: The Mayo Clinic. Recuperado el 14 de 09 de 2019

Medline Plus. (2016). Obstrucción del conducto lagrimal. Maryland: Biblioteca Nacional de Medicina de los EE. UU. Recuperado el 12 de 09 de 2019

Oftalvist. (2017). CIRUGÍA DE VÍAS LAGRIMALES. Madrid, España: autor. Recuperado el 12 de 09 de 2019

Provisu. (01 de Noviembre de 2016). Provisu. Recuperado el 12 de 09 de 2019, de Provisu: https://www.provisu.ch/es/enfermedades-mas-frecuentes/obstruccion-del-conductolagrimal.html

Salvin, J. (Febrero de 2015). KidsHealth. Recuperado el 10 de 09 de 2019, de KidsHealth: https://kidshealth.org/es/parents/tear-duct-obstruct-surgery-esp.html

Soler, J., Castillo, J., De Gregorio, M. M., \& Bescós, C. (2003). Obstrucción de vía lagrimal tratada mediante Stent lacrimonasal. Archivos de la Sociedad Española de Oftalmología, 78(6), 315-318. Recuperado el 12 de 09 de 2019, de http://scielo.isciii.es/scielo.php?script=sci_arttext\&pid=S0365-66912003000600005 